\title{
Role of Biotransformation Studies in Minimizing Metabolism-Related Liabilities in Drug Discovery
}

\author{
Yue-Zhong Shu, ${ }^{1,3}$ Benjamin M. Johnson, ${ }^{1}$ and Tian J. Yang ${ }^{2}$
}

Received 12 October 2007; accepted 13 February 2008; published online 13 March 2008

\begin{abstract}
Metabolism-related liabilities continue to be a major cause of attrition for drug candidates in clinical development. Such problems may arise from the bioactivation of the parent compound to a reactive metabolite capable of modifying biological materials covalently or engaging in redox-cycling reactions leading to the formation of other toxicants. Alternatively, they may result from the formation of a major metabolite with systemic exposure and adverse pharmacological activity. To avert such problems, biotransformation studies are becoming increasingly important in guiding the refinement of a lead series during drug discovery and in characterizing lead candidates prior to clinical evaluation. This article provides an overview of the methods that are used to uncover metabolism-related liabilities in a preclinical setting and offers suggestions for reducing such liabilities via the modification of structural features that are used commonly in drug-like molecules.
\end{abstract}

KEY WORDS: bioactivation; biotransformation; CYP; drug discovery; glutathione; metabolism.

\section{INTRODUCTION TO METABOLIC LIABILITIES IN DRUG DISCOVERY}

A major aim in drug discovery is the identification of safe and efficacious medicines that can be administered according to convenient once- or twice-daily dosing regimens. Since metabolic processes influence many other parameters that are relevant in this regard, including bioavailability, systemic clearance and toxicology, issues relating to drug metabolism are important in the selection of viable drug candidates. Substantial emphasis was placed in previous decades on the advancement of knowledge and the development of new technologies in the areas of absorption, distribution, metabolism and excretion (ADME) to assist in the identification of compounds with drug-like characteristics early in discovery. These improvements included the widespread adoption of animal models for the assessment of oral exposure and drugmetabolism screening assays for the measurement of parentcompound disappearance in liver microsomes as a proxy for hepatic CYP activity. Recently, evidence has emerged that these investments have paid off in helping to reduce the number of compounds discontinued due to unacceptable pharmacokinetic properties (1). There remains much progress to be made, however, in reducing attrition on account of other

\footnotetext{
${ }^{1}$ Department of Pharmaceutical Candidate Optimization, BristolMyers Squibb Company, 5 Research Parkway, Wallingford, Connecticut 06492, USA.

${ }^{2}$ Department of Non-Clinical Safety, Hoffmann-La Roche Inc., 340 Kingsland Street, Nutley, New Jersey 07110, USA.

${ }^{3}$ To whom correspondence should be addressed. (e-mail: YueZhong. Shu@bms.com)
}

biotransformation-related liabilities. The identification of bioactivation pathways that might produce toxicity and the prediction of drug-drug interactions that might lead to alterations in the pharmacokinetic profiles of co-administered agents continue to be major challenges in drug discovery (2-4). It is believed that similar technological advancements adopted recently will allow such properties to be identified early in drug discovery, streamlining the selection of lead candidates and ultimately leading to the development of higher quality drugs.

\section{Reactive Metabolites}

One way that a chemically reactive metabolite may exert adverse effects is by inactivating the same drug-metabolizing enzyme that catalyzes its formation, a situation that applies especially to compounds that are highly reactive or exhibit favorable binding interactions inside the catalytic pocket. Such an event may raise the exposure of either the parent compound or another drug (i.e. probe substrate) that relies on the same enzyme for clearance (5). The magnitude of the change in exposure depends on both the inactivation kinetics $\left(K_{\mathrm{I}}, k_{\text {inact }}\right.$, partition ratio $r$ ) and the extent of involvement of the enzyme in the elimination of the probe substrate (6-9). Another path to adverse effects involves the escape of the reactive metabolite from the catalytic site. This event requires the metabolite to be stable enough to resist both solvolysis and covalent binding to functional groups lining the surface of the enzyme. Reactive metabolites that leave the pocket may react with GSH covalently or, in the case of quinoid metabolites, lower GSH levels indirectly via the cycling of reactive oxygen species; either scenario represents a potential source of oxidative stress for the cell that may lead to various 
adverse outcomes. Alternatively, direct covalent modification of a large amount of protein in the cell may result in dysfunction, apoptosis or necrosis. At the organism level these changes may manifest as acute organ toxicity, especially hepatoxicity. Toxicities of the kidneys, lungs, adrenal glands, and skin have also been reported in cases involving either non-hepatic bioactivation or the transport of reactive metabolites through the circulatory system. Idiosyncratic adverse drug reactions (IADRs) are another concern in regard to the adverse effects of reactive metabolites, which may act as haptens in conjunction with endogenous proteins (10-13). In certain cases, oxidative stress and extensive covalent protein binding may represent "danger signals" that exacerbate the immunological response to these antigenic complexes and magnify the toxicity of the reactive metabolite. Although it is not possible using present technology to predict which reactive metabolites will act as haptens and which will not, it has been noted that compounds that are administered in low doses carry a reduced risk of IADRs.

\section{METHODS FOR THE ELUCIDATION OF BIOTRANSFORMATION-RELATED LIABILITIES}

Generally speaking, a select few possible causes have been recognized for the onset of undesired biological effects associated with drug metabolites. One possibility is the bioactivation of the parent compound to a reactive metabolite, which may be capable of either modifying biological material covalently or participating in redox-cycling reactions (14) leading to the formation of other toxicants. Many such metabolites have been observed to possess intermediate stability (15) or commandeer transport systems within the circulatory system (16), allowing them to exert effects that are remote from the site of formation. Alternatively, interactions between metabolites and nearby drug-metabolizing enzymes may alter metabolic clearance in a time-dependant fashion or affect the kinetics of co-administered pharmaceutical agents. Noncovalent off-target binding, possibly leading to adverse pharmacological events, is an infrequent occurrence (17) but is potentially problematic for drugs that are converted extensively to metabolites that enter systemic circulation and/or distribute into tissues. The need to predict such biotransformation-related liabilities, and to select those compounds with the greatest chance of clinical success, has led to the development of methods that can be used to identify problematic compounds in the discovery stage. The following section describes some of the most helpful procedures that are used commonly toward this end.

\section{In Vitro Bioactivation Studies}

Trapping with Glutathione and Other Nucleophiles. Experiments seeking to identify stable conjugates as surrogates for the formation of short-lived, electrophilic metabolites have become widely used in the pharmaceutical industry. Many variations on these experiments have been developed over recent years, but common elements appear to include the addition of a low-molecular-weight nucleophile to a preparation containing a test article and a drug-metabolizing homogenate (e.g. liver microsomes or S9 with necessary cofactors included), followed by an incubation period and characterization using LCMS-MS of any conjugates that are formed. The endogenous tripeptide glutathione (GSH) serves as a sensible trapping agent in this context, since it is involved in both the toxication and detoxication of certain xenobiotics in the liver. In vitro, concentrations of GSH in the range of $5-10 \mathrm{mM}$ are often used to mimic this hepatic environment. The selective detection of GSH conjugates within complex mixtures is usually achieved by positive-ion electrospray mass spectrometry and neutral-loss scanning for components that liberate the $\gamma$-glutamyl moiety (129 Da) of GSH, one of several groups released readily during mass spectrometry and collision-induced dissociation (CID) (18). Alternative screening approaches have also been proposed. For example, the use of precursor-ion scanning for the charged $\gamma$-glutamyl group $(\mathrm{m} / \mathrm{z}, 130)$ is similarly applicable and also allows the detection of biprotonated GSH conjugates, such as bis-glutathionyl metabolites or large-molecular-weight GSH conjugates that typically give neutral losses of $64.5 \mathrm{Da}$ instead of 129 Da during CID (19). Also in use are several "GSH-like" trapping agents, which are intended to retain the nucleophilic reactivity of GSH while incorporating other properties to facilitate analysis or quantification of the resulting conjugates. GSH analogs that have been dansylated (20), esterified (21), or labeled with either stable (22) or radioactive isotopes are all examples of these modified trapping agents which can be analyzed readily using tandem mass spectrometry and other techniques such as fluorescence detection or scintillation counting.

Whereas GSH and similar thiols are broadly reactive toward electrophilic substrates, occasionally they may be ineffective in trapping reactive metabolites from a few select classes. For example, the oxidation of an alkylamine may involve a spontaneous loss of water leading to the formation of an iminium ion that is reactive toward "hard" nucleophiles but not toward GSH. To ensure that the potential reactivity of these metabolites does not go unnoticed, a drug-metabolizing system may be supplemented with a cyanide salt instead of GSH to give conditions that are favorable for the formation of cyano adducts (23). Because of the highly toxic nature of solutions containing cyanide, the use of these methods requires a strong commitment to careful handling and proper disposal. However, even if cyanide is not added to these solutions directly, a cyano adduct may still be observed if the test article is pre-dissolved in acetonitrile and the enzymatic preparation (e.g. liver microsomes) is capable of generating small quantities of cyanide in vitro. Like a GSH adduct, a cyano adduct may be identified readily using positive-ion electrospray (or APCI) tandem mass spectrometry, often exhibiting a mass gain of $25 \mathrm{Da}$ over of the parent compound and a neutral loss of HCN (27 Da) during MS/MS with CID.

In addition to the use of cyanide and GSH-related compounds as trapping agents, the use of amines has also been reported for the trapping of acylhalides and glyoxal groups (3). However, screening applications where these agents are added to liver microsomes for the trapping of oxidation products do not appear to be used widely.

Covalent Binding. The covalent-binding experiment represents a complementary approach to measuring rates of reactive-metabolite formation in liver microsomes, with correlations between rates of covalent binding and GSHconjugate formation having begun to emerge (24). Experimental procedures for this experiment, which have been 
outlined previously in detail, (25) require the synthesis of test articles that are labeled with $\beta$-emitters such as ${ }^{3} \mathrm{H}$ or ${ }^{14} \mathrm{C}$ and are therefore substantially more resource-intensive than the trapping experiments described above. Briefly, these test articles (usually $10 \mu \mathrm{M}$ ) are incubated with liver microsomes (1 mg protein/ml), NADPH, and either GSH or cyanide (optional) for sufficient time to give the desired metabolic turnover, and then protein is precipitated, washed and dissolved for scintillation counting. A reduction in the level of covalent binding in the presence of GSH or cyanide may implicate certain structural features of the reactive intermediate and provide evidence for its escape from the catalytic site. Researchers at Merck \& Co. have proposed a binding level of 50 pmol mg protein ${ }^{-1} \mathrm{~h}^{-1}$ as a conservative threshold for an acceptable level of covalent binding for a drug candidate. This number was based on (a) levels of protein adducts observed in livers following bioactivation-mediated necrosis, and (b) a 20 fold safety margin, below which such necrosis would presumably be unlikely (25). Although Merck has reported some success in using this approach to identify problematic compounds in its discovery efforts, both the cost-benefit relationship of maintaining a radiosynthesis group to support covalent-binding studies and the utility of the $50 \mathrm{pmol} \mathrm{mg}^{-1} \mathrm{~h}^{-1}$ threshold in deciding whether to advance compounds into development continue to be topics of debate in the greater drug-metabolism community. On the latter point, additional considerations might provide a context for evaluating the potential liability associated with elevated levels of covalent binding, such as the potential for bioactivation in vivo, the clinical indication and dose, and the availability of preliminary toxicological findings.

Mechanism-Based CYP Inhibition. During multi-drug therapy, a therapeutic agent that is also an inhibitor of a CYP (or other drug metabolizing enzyme) may have the potential to affect the pharmacokinetics of co-administered agents that rely on the same enzyme for metabolic clearance (26). Hence, inhibitors of CYP3A and other enzymes that are involved in the clearance of a wide variety of substrates are usually flagged in drug discovery and precluded from advancement into development. Biotransformation studies may play a role in the identification of such compounds in certain cases. For example, the formation of a reactive metabolite may lead to the covalent modification the same CYP enzyme involved in catalysis, leading to irreversible inhibition of the enzyme (27). Such behavior can usually be attributed to chemical structures that also have a proclivity toward bioactivation and the formation of GSH and protein conjugates (e.g. alkynes, thiophenes, halogenated alkenes) (28). However, the presence of GSH may fail to prevent the onset of irreversible inhibition, since binding may occur before the substrate has a chance to leave the active site. A separate set of experiments is therefore necessary to determine whether a compound of interest may carry the liability of mechanism-based inhibition.

Studies to evaluate mechanism-based CYP inhibition usually involve pre-incubation of a test article (at multiple concentrations) in liver microsomes or recombinant CYPs, with and without NADPH, followed by the addition of a probe substrate whose rate of disappearance is then measured. Midazolam and testosterone are common probe substrates used to evaluate effects on CYP3A4. Test articles that reduce the rate of consumption of the probe substrate with respect to the control that is pre-incubated without NADPH are usually studied further. For example, dialysis experiments can be used to investigate the restoration of CYP activity following removal of unbound material, or spectrophotometric studies of CYP structure can be used to determine whether the hemeprotein has been modified. Methods for these studies have been described (29-32).

Reactivity and Rearrangement of Acyl Glucuronides. The formation of acyl glucuronides is a matter of concern in biotransformation studies on account of the potential for these metabolites to react covalently with other biological molecules including proteins. These reactions have been implicated in severe toxicity, resulting in the withdrawal of multiple drugs from the marketplace in the USA and other countries (33). In the reaction of acyl glucuronides with nucleophilic substrates, the sugar moieties of $O$-1-acyl glucuronides may either be displaced directly by nucleophilic amino acids or may first undergo acyl migration to $O$ - $n$-acyl derivatives, where $n$ may correspond to carbon atoms 2, 3, or 4 of the glucuronide moiety. In recent years, methods have been introduced to monitor the kinetics of these processes and to allow comparisons among acyl glucuronides with respect to reactivity. As a caveat it should be noted that direct relationships between the reactivity of acyl glucuronides and the severity of organ toxicity have not been demonstrated.

One approach in the assessment of acyl-glucuronide reactivity involves the trapping of an activated sugar moiety with a protein surrogate. For example, the Lys-Phe dipeptide has been shown to react with rearranged acyl glucuronides via Schiff base formation (34). The rates of these reactions appear to correlate well with those of acyl migration, suggesting that acyl glucuronides that undergo rearrangement are more reactive toward Lys-Phe than those retaining their original $O-1$-acyl connectivity. Hence, this technique could be applied to rank-order compounds within a chemical series based on the reactivity of their respective acyl-glucuronide conjugates. NMR spectrometric methods have also been introduced to monitor the stability of acyl glucuronides and elucidate the structures of their degradation products (35-37). The former task is accomplished in part by analyzing the resonance signals associated with the anomeric proton of the $O$-1-acyl glucuronide as a function of time. As would be expected, the disappearance of these signals follows firstorder kinetics, allowing the calculation of a $t_{1 / 2}$ as a measure of reactivity. Additional information about the structures of rearranged products is also available by inspection of the NMR data following an extended incubation period.

\section{Biotransformation Modeling in Silico}

The identification of metabolites is a resource-intensive process, both in terms of the time required to carry out biotransformation studies and the need for the scientist who interprets the LC-MS/MS data to be highly trained. This leads to both human and instrumental biases, especially in cases where metabolites are not radiolabeled and are hidden within complex biological matrices that may suppress ionization or 
contain isobaric interferences. In order to accelerate metabolite identification and provide a check against these biases that might cause unusual or low-level metabolites to be overlooked, informatics tools have been developed for data mining and interpretation. Anari and co-workers described the metabolic profiling of indinavir with an approach combining data-dependent LC-MS/MS and knowledge-based metabolite predictions that were generated using a substructure similarity search of the MDL Metabolite Database (38). Several other programs have been developed for this purpose (39-44), including an approach co-developed at Bristol-Myers Squibb for LC-MS/MS data mining of potential metabolites. As shown in Fig. 1, the tool named "Metabolite Profiler" combines a comprehensive collection of metabolic modifications (reaction table) with structure-specific metabolite predictions using METEOR (Lhasa Ltd) (45). The Profiler calculates the molecular masses of predicted metabolites, removes duplicates, and automatically generates a result file that can be imported into data-analysis programs (e.g. MetWorks). The imported result file is then used to generate customized extracted-ion chromatograms for data mining and identification of potential metabolites based on anticipated mass-to-charge ratios. This routine has been incorporated into the workflow for biotransformation studies, proving to be useful in broadening the coverage of potential metabolites and providing a check against human and instrument-related biases.

\section{Animal Models and the Study of Metabolic Fate}

Use of Mechanism-Based Inhibitors in the Elucidation of Toxicological Mechanisms. Whereas mechanism-based inhibitors are usually avoided in multi-drug therapy for the reasons described above, they can be useful tools in determining whether a toxicological event in vivo is the result of exposure to a compound or its metabolites. The suicide inhibitor 1-aminobenzotriazole (ABT) is the most widely used tool in this regard. ABT undergoes bioactivation to a reactive intermediate that alkylates most CYPs, resulting in their inactivation (46). Thus, the pretreatment of animals with ABT during a toxicology study (a dose of $50 \mathrm{mg} / \mathrm{kg}$ administered $2 \mathrm{~h}$ before administration of the test article is a common practice $(47,48)$ ) may reduce the severity of adverse events, but only if such events are mediated by a toxic metabolite and not the parent compound itself. This approach has been used to implicate metabolites in the onset of hepatotoxicity in mice following administration of furosemide, (49) and nephrotoxicity in rats following treatment with
[2,3- $\left.{ }^{14} \mathrm{C}\right]-N-(3,5-$ dichlorophenyl)succinimide (50). In both of these cases, toxicity of the target organ correlated positively with covalent binding and was reduced in animals pretreated with ABT.

Absorption, Distribution, Metabolism and Elimination (ADME) Studies Using Bile-Duct-Cannulated Animals. The characterization of promising compounds in drug discovery usually includes a series of studies to ascertain the main metabolic and disposition pathways in animals. When the results of these studies are considered alongside in vitro biotransformation data, some initial predictions can be made as to which clearance pathways might be the most important clinically, and which metabolites detected in human liver microsomes (or hepatocytes) will also be represented during toxicological assessments in animals. These predictions can help prepare an organization for upcoming non-clinical toxicology studies during drug development, in light of FDA guidelines for the evaluation of metabolites in drug safety (51).

Biotransformation studies using bile-duct-cannulated (BDC) animals are advantageous in this regard, allowing metabolic profiling across species and offering insight into the extent of formation in vivo of metabolites of interest, such as GSH conjugates or acyl glucuronides, which may indicate bioactivation (52). Studies in BDC animals can also provide information on mass balance, absorption, tissue distribution, or elimination of a drug, depending on study design. Mass balance is obtained typically by dosing ${ }^{14} \mathrm{C}$-labeled compound and then quantifying radioactivity present in bile, urine, and feces over a collection interval of sufficient length to allow extensive elimination of the drug and its metabolites (53). Radioactivity remaining in the tissues following the collection interval can also be quantified either via combustion and liquid-scintillation counting of individual organs or using whole-body autoluminography. Although studies using radioactive test articles are typically carried out with the aim of recovering high percentages of the doses administered, there are differing perspectives within the industry as to what criteria are most useful in deciding whether a study has fulfilled its objectives and whether studies achieving only moderate recovery should be repeated (54).

The design of a study using BDC animals, with consideration given to routes of administration and the selection of proper controls, determines which of the ADME parameters can be estimated in the interpretation of the data. For example, a comparison of plasma concentrations in two groups dosed via portal-vein infusion and intravenous infusion, respectively, provides an estimate of hepatic extraction

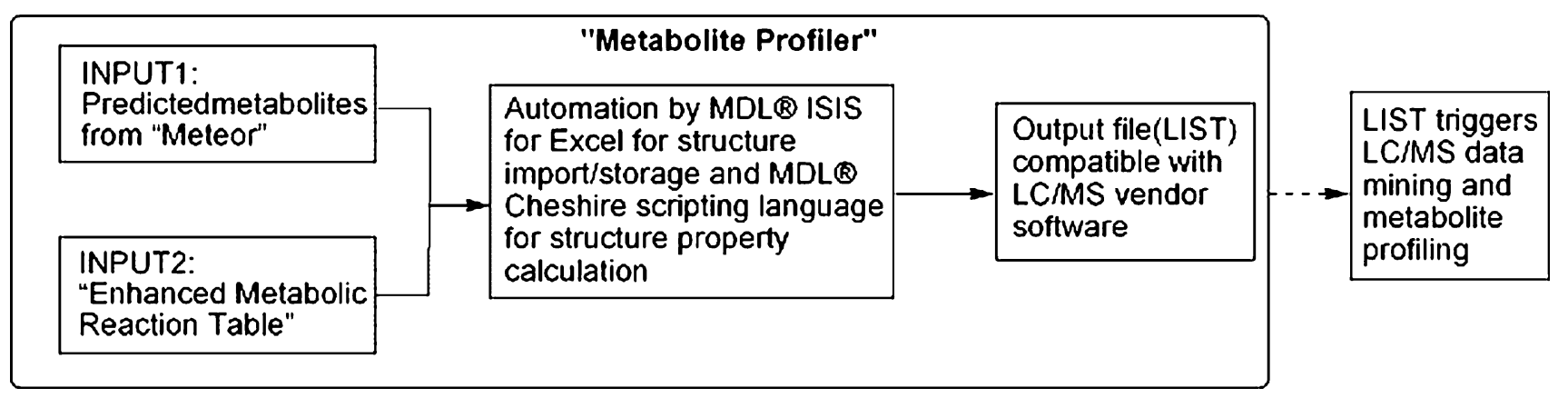

Fig. 1. Procedure for the identification of metabolites using Metabolite Profiler 
during first-pass metabolism. A comparison of fecal contents following oral and intravenous dosing provides information on the extent of absorption from the gastrointestinal tract and the possible involvement of P-glycoprotein and other transporters in disposition. Similarly, differential absorption in BDC and non-cannulated animals can help distinguish between enterohepatic recirculation and discontinuous absorption as possible causes for inflections in plasma concentrations over time, as illuminated during pharmacokinetics studies. It is therefore important to consider the questions to be answered during such a study before selecting methods for the administration of the test article.

Covalent Binding in Vivo. In addition to the methods described above pertaining to covalent-binding experiments in vitro, similar experiments have been described to characterize binding levels in animals. In both cases, compounds labeled with ${ }^{14} \mathrm{C}$ are the substrates of choice because they do not experience losses of radioactivity in solution, as is sometimes the case with ${ }^{3} \mathrm{H}$ tracers. However, it is still important to install the ${ }^{14} \mathrm{C}$ label in a position that does not undergo metabolism; thus, biotransformation studies are often carried out in advance to characterize metabolic pathways in the animal of interest before labeled-compound synthesis is undertaken. To prepare samples, animals are dosed with radioactive compound, sacrificed after a time based on pharmacokinetic parameters, and the organs of interest are removed. These organs usually include the liver, where concentrations of CYPs and other drug-metabolizing systems are high, although marginal levels of binding in other organs such as lung and kidney have also been reported (55). Following homogenization of these organs and centrifugation into subcellular fractions, the methods used for the washing of protein and the counting of radioactivity resemble those used during covalent-binding studies in vitro.

Distribution Studies Using Mass Spectrometric Imaging. Recent advances in matrix-assisted laser desorption/ionization mass spectrometry (MALDI-MS) have enabled the creation of "maps" showing the spatial distribution of drugs and their metabolites in tissue sections of either organs or whole animals. This technique has certain advantages over whole-body autoradiography, since it can be performed using a non-radiolabeled drug and has the capacity to distinguish among drug-related compounds based on mass-to-charge ratios. Potentially, this latter feature allows for the creation of a separate map for the distribution of each metabolite in a mouse or other small animal. Furthermore, a linear relationship between the concentration of a compound in a sample and the intensity of a MALDI-MS signal can be defined over a wide dynamic range, allowing quantitative information to be obtained on any ionizable analyte (parent compound, metabolite, or endogenous component) for which a synthetic standard is also available. This is a particularly useful tool in addressing situations where a metabolite is known to have an effect on a particular organ or substructure (e.g. a seizurogenic metabolite in the brain, an ion-channel inhibitor in the heart, etc.), providing information on the distribution and concentration of the compound within the organ of interest. Review articles and other useful descriptions of MALDI-MS imaging are available in the literature (56-58).

\section{Genetic Modification}

Emerging techniques such as RNA interference (RNAi) and the use of genetically modified (e.g. knockout, transgenic) animals are being applied increasingly in the study of drug metabolism. RNAi can be used to knock down the expression of a gene for a drug-metabolizing enzyme or transporter in a post-transcriptional manner, allowing the role of such proteins in the metabolism of a xenobiotic substrate to be characterized specifically (59). During drug discovery and development, this technique may be particularly useful in helping to resolve a toxicological issue by allowing an adverse event in an animal to be ascribed to a species-specific structure or process. Although few such examples have been published, a similar approach was used recently to show that knockdown of the gene encoding the $\alpha$-synuclein protein resulted in the protection of human neurons against the neurotoxin $\mathrm{MPP}^{+}$(60). Animals that are engineered to express specific human proteins are also under development in an attempt to produce models that facilitate more accurate predictions of human metabolism. Reviews of this topic are available in the literature $(61,62)$.

\section{MOLECULAR DESIGN AND THE AVOIDANCE OF METABOLISM-RELATED LIABILITIES}

The failure of drug candidates can often be attributed to the toxicological effects of particular functional groups that undergo bioactivation; thus, reactive metabolites are generally considered within the pharmaceutical community to represent potential liabilities in the development of a drug. Recently, increasing emphasis has been placed on the detection and identification of chemical markers of bioactivation, including GSH conjugates, and this effort has been assisted by improvements in the sensitivity and selectivity of spectrometric instrumentation. As a result, many structurally diverse and unusual GSH conjugates have been identified despite relatively low quantities in biologic matrices. Most of the bioactivation sequences leading to these metabolites can be rationalized in the context of basic CYP-mediated oxidations and rearrangements, some of which are affected by neighboring or sterically proximal functional groups in the substrate molecule.

When a reactive metabolite is found, an investigation of the underlying bioactivation mechanism may lead to a more complete understanding of the potential metabolic liability. Often these studies lead to the identification of problematic structural features to be avoided in the evolution of a lead series, such as those summarized in Fig. 2 that lead to the formation of GSH or cyanide adducts. In light of the increasingly large volume of literature in this area, including review articles that cover related topics $(3,25,63)$, this section is intended to be illustrative rather than comprehensive, and the structure types to be discussed are limited primarily to druglike molecules. As a point of differentiation from published reviews, this section also focuses on strategies to avert bioactivation in certain chemical series by suggesting appropriate substitutions to functional groups in representative compounds. This approach to drug discovery extends the concept of bioisosteric replacement beyond its typical application in optimizing the physicochemical properties and biological activities of test compounds, and it requires a close 
1. Displacement reactions

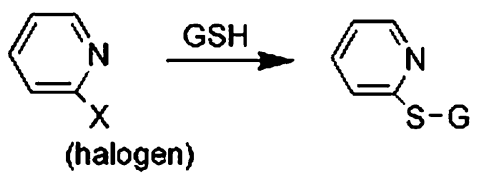

\section{Michael reaction additions}<smiles>[R]C=CC(C)=O</smiles><smiles>[R]Nc1ccc(O)c(S(=O)(=O)[O-])c1</smiles>

\section{Reactions via epoxides or arene oxides}
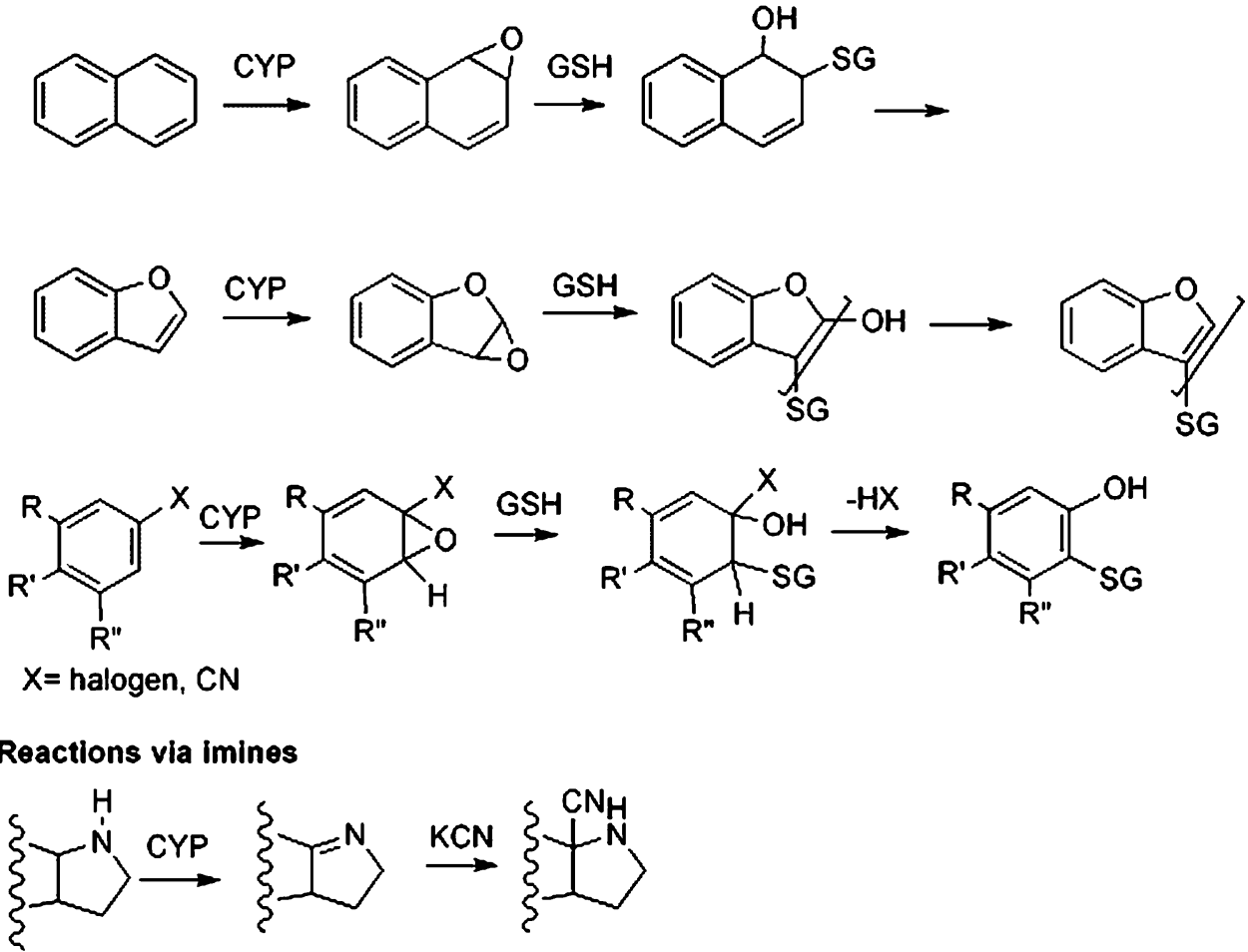<smiles>[SiH3][SiH3]</smiles><smiles>[R]C1=CC([X])(O)C(C)([S-])C([R7])=C1[R]</smiles><smiles>[R]c1cc(O)c([SeH])c([R])c1[R]</smiles>

Fig. 2. Common bioactivation pathways leading to glutathione or cyanide adducts

partnership between biotransformation scientists and medicinal chemists in managing the evolution of a chemical series.

\section{Amines and Azacycles}

Cyclic and acyclic secondary and tertiary amines and azacycles are used widely as functional groups in drug-like molecules despite a proclivity toward bioactivation to electrophilic iminium ions and/or carbinolamines. These two types of electrophiles may have distinct mechanisms of formation but are usually assumed to co-exist with each other in a hydrolytic equilibrium. Decomposition of these products via $N$-dealkylation is a possibility that may lead to the elimination of the carbinol-bearing moiety and sometimes to intra-molecular rearrangement. Alternatively, there is evidence that iminium ions from nitrogen-bearing heterocycles may be oxidized further to stable lactams in the presence of cytosolic aldehyde oxidases. The reactivity of iminium ions toward GSH is sometimes obscured by the instability of the resulting conjugate under laboratory conditions; thus, a cyanide salt is often used to supplement microsomal preparations such that they may be trapped as meta-stable cyano 
adducts instead. Such reactions have led to the proposal that iminium ions might react with nucleophilic sites within cellular macromolecules and contribute to the pharmacology or toxicology of aliphatic amines (64).

The alkylation of DNA by imines has been implicated in the antitumor pharmacology of multiple DNA-acting cytotoxic natural products, including ecteinascidin 743 (65), cyanocycline (66), doxorubicin (67) and barminomycin (68). The corresponding imines, accessible either directly or via bioactivation, have been reported to react covalently with DNA in a base-specific manner. (Fig. 3).

Extensive metabolic, biochemical, and toxicological investigations have established that the neurodegenerative properties of the nigrostriatal toxin 1-methyl-4-phenyl-1,2,3,6tetrahydropyridine (MPTP; Fig. 4) are mediated in a tissueselective manner by the pyridinium metabolite $\mathrm{MPP}^{+}$, a product of catalysis by monoamine oxidase $\mathrm{B}$. Whereas covalent binding with macromolecules is not implicated directly, $\mathrm{MPP}^{+}$selectively inhibits the activity of complex I of the mitochondrial electron transport chain. The depletion of ATP that follows from this inhibition is considered to be the principal cause of cell death.

Multiple cyclic amines, such as $N$-benzylcyclopropylamine, phencyclidine, and $(S)$-nicotine (Fig. 4) were reported to undergo bioactivation to iminium intermediates, a pathway associated with covalent binding to macromolecules. Covalent binding during the metabolism of $(S)$-nicotine is reduced by cyanide, and studies have shown that $N$-benzylcyclopropylamine and phencyclidine cause CYP inactivation during metabolism. The iminium metabolite of $N$-benzylcyclopropylamine inactivates these enzymes by covalent modification of the active site, whereas phencyclidine is not classified as a mechanism- based inactivator since its reactive intermediate is believed to escape the active site.

Pyrrolizidine alkaloids (PAs) such as reddiline, which occur naturally in flowering plants from the Boraginaceae, Compositae and Leguminosae families, have been shown to be genotoxic and tumorigenic in experimental animals. CYPcatalyzed $N$-oxidation of these compounds results in the formation of non-toxic metabolites that are highly polar and easily excreted. However, CYP also catalyzes dehydrogenation to unstable pyrroles that may undergo further $O$ dealkylation to give DHP (69) (Fig. 4). Both DHP and the PA pyrroles can react with DNA and other macromolecules via the formation of carbonium intermediates (70). Since these intermediates are highly reactive, their effects in vivo are usually confined to the site of formation in the liver and other CYP-expressing tissues. Thus, hepatotoxicity is the most prominent effect of PA administration in animals.

The structural modification of amines that are prone to bioactivation generally focuses on attenuating the extent of formation of the iminium species. Lowering the substitution on the nitrogen atom, for example replacing a tertiary amine with a secondary amine, usually stabilizes the nitrogen against electron abstraction by raising the energy of the transition state en route to the radical intermediate. The nitrogen can also be stabilized against CYP-mediated metabolism by introducing steric hindrance on functional groups nearby or installing alkyl or halogen groups at the $\alpha$ - or $\beta$ - carbons. In the case of 5- or 6-membered cyclic amines, it may also be helpful to fuse these structures into heterocycles or reduce the number of atoms in the ring (e.g. replace a pyrrolidine ring with an azetidine moiety) to overcome a metabolic liability (Fig. 5).

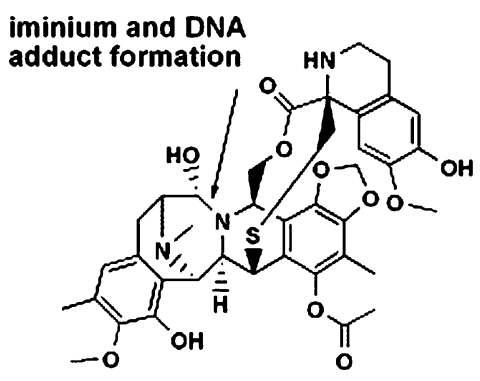

ecteinascidin 743

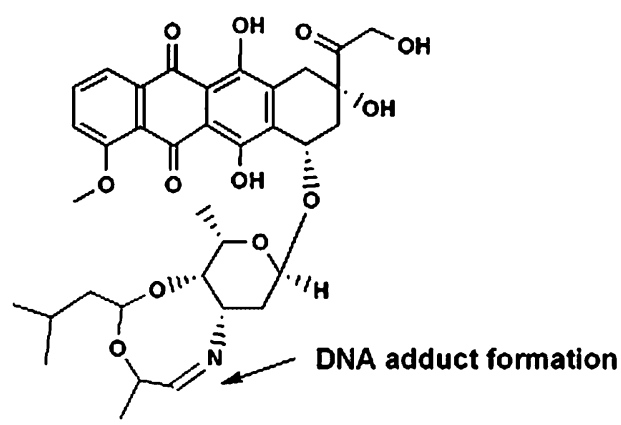

barminomycin (imine form)

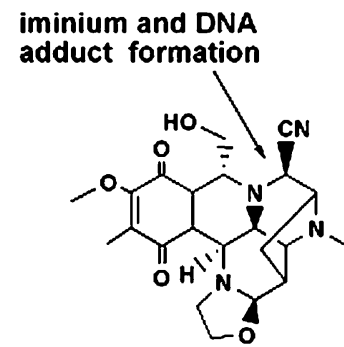

cyanocycline

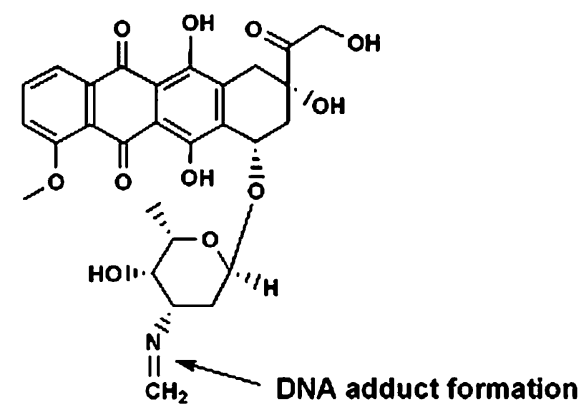

formaldehyde-activated form of doxorubicin

Fig. 3. Examples of cytotoxic natural products that form DNA adducts via imine or iminium intermediates 
<smiles>CN1CC=C(c2ccccc2)CC1</smiles><smiles>c1ccc(CNC2CC2)cc1</smiles>

MPTP<smiles>c1ccc(C2(N3CCCC3)CCCC2)cc1</smiles>

phencyclidine<smiles>CN1CCCC1=Cc1ccccc1</smiles>

(S)-nicotine<smiles>C=C(CO)C/C(=C/C)C(=O)OC1CCN2CC=C(COC(=O)[C@](C)(O)C=O)C12</smiles>

riddelline

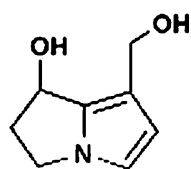

DHP

Fig. 4. Examples of secondary and tertiary amines that undergo bioactivation

A few examples of how these strategies can be incorporated into a drug-discovery workflow were provided recently. In one such case, a piperazine-containing compound had been deemed unviable as a drug candidate because of high

covalent binding ( $\sim 300 \mathrm{pmol} \mathrm{eq} / \mathrm{mg}$ protein $)$ and timedependent CYP3A4 inactivation in human liver microsomes. However, structural modifications to its lactam- and $\alpha, \alpha^{\prime}$ dimethyl-substituted derivatives were employed to minimize oxidation of the piperazine nitrogen and the formation of a reactive iminium moiety, giving rise to new analogs that avoided these liabilities (71). In another case, the discovery of sitagliptin, a dipeptidyl peptidase IV (DPP-4) inhibitor, was facilitated by structural modifications to a series of piperazine-containing compounds that resulted in lower metabolic clearance. In order to reduce the level of oxidative metabolism among early DPP-4 inhibitors containing the piperazine, bicyclic derivatives including imidazolopiperazines and triazolopiperazines were prepared and evaluated in various metabolism models, including BDC animals. This iterative lead optimization effort yielded improvements in the oral bioavailability of newer compounds and ultimately led to the discovery of sitagliptan, which was selected from the triazolopiperazine series for further development and was approved by the US FDA in 2006 for the treatment of type 2 diabetes (72).

\section{Aromatics}

Arylamines or anilines have long been associated with chemical carcinogenesis, and reports have indicated that the genotoxicity of arylamines is tied to metabolic activation, proceeding initially by $N$-hydroxylation of the primary amine

\section{Reduce imine formation}

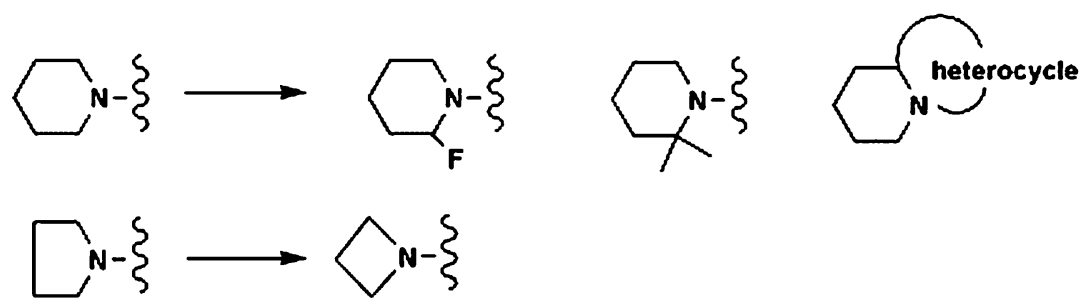

\section{Bioisosters of olefin and activated olefin}<smiles>[Z6]C=CC(C)=O</smiles><smiles>CC=CC(C)(F)F</smiles>

\section{Bioisosters of phenol}<smiles>Oc1ccccc1</smiles>

phenol<smiles>CO[Sb]([O-])([O-])Nc1ccccc1</smiles>
sulfonamide<smiles>[X]c1ccccc1NC</smiles><smiles></smiles>

$X=C, N$

\section{Bioisosters of carboxylate}<smiles>O=C(S)SCCS</smiles>

carboxylic acid

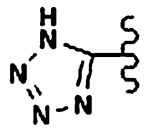

tetrazole<smiles>[R]NC(C)=O</smiles>

amide

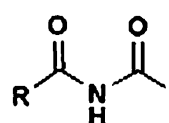

imide
$\mathrm{MeO}_{2} \mathrm{~S}$<smiles>CC(=O)N[AsH]</smiles>

sulfonamide

Fig. 5. Bioisosteric alternatives to selected functional groups 
$(73,74)$. The reactive $N$-hydroxylamine metabolites can either undergo oxidation to more reactive nitroso intermediates or conjugations to $\mathrm{N}$ - $\mathrm{O}$-sulfates or $\mathrm{N}$-O $\mathrm{O}$-acetates, effective leaving groups that may lead to highly reactive nitrenium ions. Due to heightened awareness of the potential genotoxicity of arylamines, unprotected and oxidizable primary arylamines are not deemed to be drug-like and are rarely seen in today's drug candidates. However, latent arylamines that are chemically masked and not subject to oxidation are still used as core scaffolds in medicinal chemistry. In drug development, the potential metabolic release of a mutagenic free arylamine remains a concern for human safety, and health regulators may require monitoring of released arylamine in humans using sensitive LC-MS-MS methods, posing a hurdle to regulatory approval. Thus, it is important to assess and minimize the formation of such genotoxic metabolites before a clinical candidate is selected. To this end, non-mutagenic arylamines may be adopted as surrogates for anilines. Among masked arylamines, the risk of releasing free arylamine during hydrolytic or oxidative metabolism is largely related to the stability of the bond connecting the arylamine moiety to the rest of the molecule (Fig. 6). Additional synthetic strategies may therefore focus on forming a metabolically stable linkage while retaining any desired activity.

The potential metabolic formation of ortho or paraaminophenols from masked arylamines also represents a structure alert (Fig. 2), as they could be oxidized subsequently to quinone imines. A strategy to avert the formation of such electrophilic intermediates is to introduce electron-withdrawing groups into the ring system to block the oxidation site directly or reduce the overall electron density of the ring. As a caveat, oxidative dehalogenation has been noted during the metabolism of certain halogen-substituted aromatics, such as hexachlorobenzene (75), voriconazole (76), and DPC 963 (77) (Fig. 7), and some of these compounds go on to form GSH conjugates. The oxidations are catalyzed by CYP and may proceed either by ipso attack and sigma complex formation, or via the formation of an arene oxide (with or without an NIH shift involving the halogen atom) followed by elimination (Fig. 2) (75,76). Although the occurrence of CYPmediated oxidations of halogenated phenyls may seem at odds with the electron-withdrawing effects of halogens, this reactivity may be attributable to the mesomeric properties of

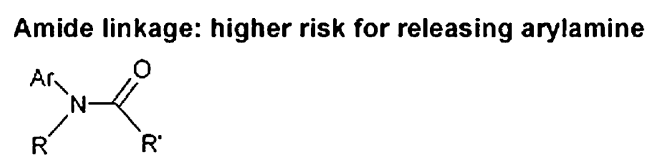

Alkyl or carbamate linkage: moderate risk for releasing arylamine<smiles>[R]CN([R])[Al]N([R])C(=O)O[R7]</smiles>

Aryl or urea linkage: low risk for releasing arylamine<smiles>[R]NC(=O)N([R])[Al]</smiles>

Fig. 6. Masked arylamine linkages and relative risk for enzymemediated arylamine release halogen atoms rather than their electron-withdrawing effects. Additionally, aromatic halogenation often leads to increased lipophilicity, which may augment both tissue penetration and affinity for the CYP catalytic site. Thus, the metabolism of halogenated aromatics depends on multiple factors and is difficult to predict.

\section{$\pi$-Rich and Related Five-Membered Heterocycles}

Five-membered $\pi$-rich heterocycles such as pyrroles, furans, thiophenes, and their benzo-fused derivatives are widely used in drug molecules. These electron rich systems share a common electronic property, with six electrons distributed over five atoms, and undergo many metabolic reactions leading to both stable and reactive metabolites (Fig. 2). A multitude of related biotransformations on 5membered heterocycles (78) and implications for potential toxicity (3) were reviewed recently and will not be discussed further. Compared to pyrroles, furans and thiophenes, ring systems containing an additional nitrogen atom such as azoles and isoazoles share the common characteristics of reduced $\pi$ electron density and a new site of basicity. Both of these properties can affect their metabolism. The placement of the nitrogen atom within the ring is also important. For example, imidazoles, oxazoles, and thiazoles are susceptible to oxidative metabolism and sometimes bioactivation, whereas isoxazoles and isothiazoles undergo reductive ring cleavage, sometimes by non-CYP enzymes. By contrast, pyrazole rings are relatively stable to either oxidative or reductive metabolism despite the presence of an isoazole linkage (78). Fivemembered heterocyles containing three or more heteroatoms, such as triazoles and oxadiazoles, have even less $\pi$-electron density than azoles and are also resistant to CYP-mediated oxidations. However, non-CYP mediated or mixed-mode pathways may present alternative routes of biotransformation for these compounds and lead to ring-scission products (78). The bioactivation of 5-membered heterocycles with two or more heteroatoms is difficult to generalize, and the assessment of their metabolic liabilities remains chemotype-, compound-, or even substitution-specific. Still, these heterocycles are widely used as bioisosteres for esters and amides and may offer vastly improved metabolic stability over such non-cyclic counterparts. A known metabolic liability of some pyrazoles, imidazoles and triazoles is heme coordination inside the CYP catalytic pocket. Known as type-II ligands, these compounds can inhibit CYP by replacing the hemewater ligand and stabilizing the iron in its low-spin state (7981). Certain alkyl-substituted heterocycles may be metabolized to imine-methide-like intermediates that alkylate the CYP active site, resulting in enzyme inactivation (82). The formation of type-II complexes may inhibit the metabolism of co-administered drugs and result in drug-drug interactions; thus, compounds capable of forming these complexes must be tested for CYP inhibition early in drug discovery.

\section{Six-Membered Heterocycles}

It is difficult to predict bioactivation reactions on 6membered heterocyles because of the widely variant electronic and steric effects of ring substituents. The reader is 
<smiles>CC(C)(C)O[Mg]O[Mg]</smiles>

hexachlorobenzene

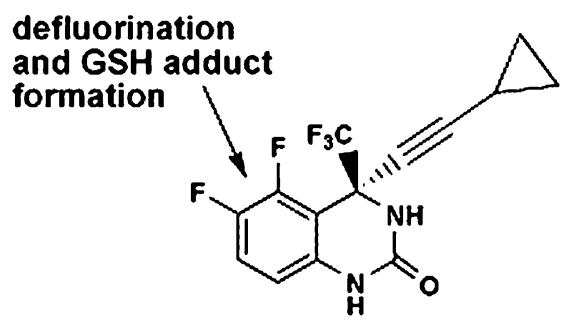

DPC 963

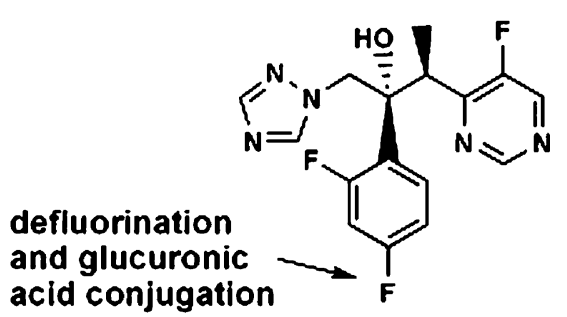

voriconazole

Fig. 7. Examples of aromatic dehalogenations

again referred to a review that provides some specific examples of bioactivation of such structures (3). Phenol rings containing $o$ - or $p$-substituted electron-donating substituents such as alkyl groups or amines are susceptible to the formation of quinoid metabolites that can act as Michael acceptors in the alkylation of cellular proteins and DNA. Electron-withdrawing substituents usually deactivate hydroquinones and cause them to be less easily oxidized, whereas the opposite is true for electron-donating groups. This observation has two important consequences: first, quinones, quinone imines, and quinone methides are successively easier to oxidize because electronegativity decreases in the order $\mathrm{O}>\mathrm{N}>\mathrm{C}(83)$, and second, the conjugation of quinones with GSH and other thiols cannot generally be viewed as a detoxication step, since the thioether is itself electron-donating and may facilitate redox cycling or covalent reactivity (84-88). The redox cycle between a quinone and hydroquinone triggers the formation of reactive oxygen species (ROS) such as superoxide, hydrogen peroxide and the hydroxyl radical, leading to cellular damage and oxidative stress (89-91). A structural alert for bioactivation vulnerability is the combination of a 6-membered heterocycle, and some combination of alkyl, amino, or hydroxyl substituents that are situated ortho or para to each other. As a structural requirement, the alkyl group must contain either a hydrogen atom or suitable leaving group on the benzylic carbon atom. The hydroxyl group is then installed metabolically, either introduced by hydroxylation or unveiled via $O$-dealkylation or hydrolysis. For example, the bioactivation of the antibacterial agent trimethoprim is mediated through a pyrimidine iminoquinone methide, which can be trapped in the presence of $N$ acetylcysteine (Fig. 8) (92).

Characterization of metabolites of a pyrazinone-containing thrombin inhibitor revealed extensive bioactivation of the pyrazinone ring system and high levels of irreversibly bound radioactivity to proteins in rats after administration of ${ }^{14} \mathrm{C}$-labeled drug (93). Metabolism of this compound in GSH-fortified liver microsomes gave two conjugates derived respectively from an imine methide and an epoxide of the pyrazinone ring (Fig. 8), the latter facilitating opening of the pyrazinone ring and subsequent rearrangements. Thus, pyrazinones may undergo bioactivation along multiple pathways.

\section{Nucleophilic Substitution Involving Glutathione}

Suitably substituted heterocyclic rings that contain aromatic nitrogens, such as 2- or 6-halopyridines, are susceptible to attack by GSH and other nucleophiles due to an activated halogen-carbon bond that leaves a partial positive charge on the carbon. In biological systems these reactions may be facilitated by glutathione $S$-transferases (Fig. 9). For example, the insecticide chlorpyrifos is metabolized by GSH-dependent substitution of the 6-chlorine on the pyridine moiety, as evidenced by abundant cysteine and mercapturate conjugates in the urine of an individual who was intoxicated acutely with this compound (94). Pyridines substituted with 2-sulfonamides may behave similarly in this regard, as the HIV-1 protease inhibitor PNU-109112 is metabolized by GST-catalyzed displacement of the sulfonamide moiety. Structure-activity studies with a variety of sulfonamides have indicated that cleavage is assisted by an electrophilic center alpha to the sulfonyl group such as a carbon atom bearing an electronwithdrawing substituent $(95,96)$ (Fig. 9). Similar nonenzymatic nucleophilic displacements of C2-substituted sulfonamides were observed on thiazoles, benzothiazoles, 1,3,4-thiadiazoles, and 1,3,4-oxodiazole rings under simulated physiological conditions $(97,98)$. The electrophilic C2 imine carbon and the presence of a good leaving group, such as a sulfonamide, are structural prerequisites for the displacement to occur (Fig. 9).

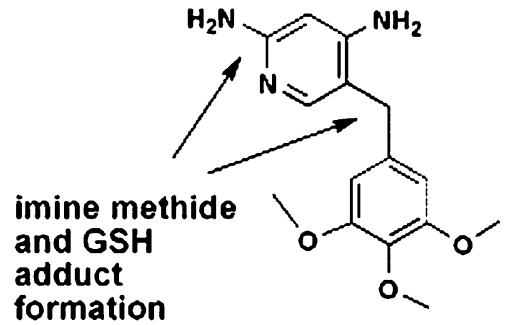

trimethoprim

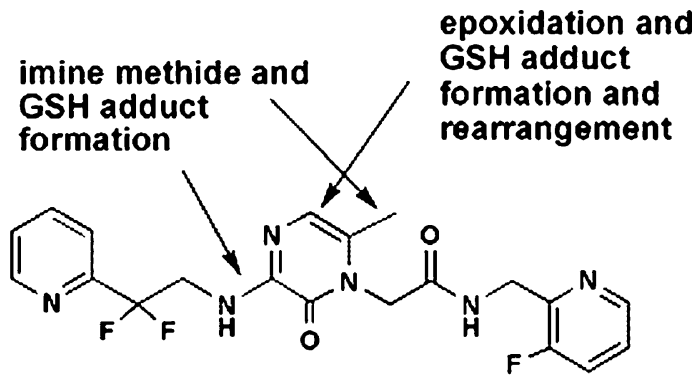

compound I

Fig. 8. Bioactivation of 6-membered heterocycles 
<smiles>CCOP(=S)(OCC)Oc1nc(S(O)(=S)=S)c(Cl)cc1Cl</smiles>

chlorpyrifos<smiles>[3H]NS(=O)(=O)c1ccc([SeH]S(=O)(=O)c2ccc(C#N)cn2)cc1</smiles>

PNU-109112

partial structure
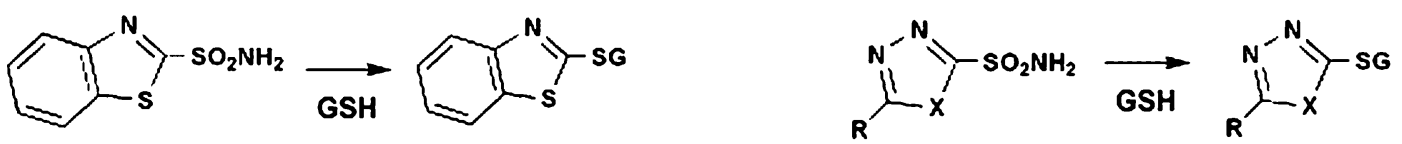

$X=S, 1,3,4$-thiadiazole

$X=0,1,3,4$-oxadiazole

Fig. 9. Nucleophilic aromatic substitutions involving glutathione

\section{Olefins and $\alpha, \beta-$-Unsaturated Carbonyls}

The epoxidation of olefins is a well known bioactivation pathway, as reactive products have been demonstrated to mediate toxicity via reaction with nucleophilic groups in macromolecules. The reactivity of these products is enhanced via 1,2- or 1,4-Michael addition if the olefin is a part of an $\alpha, \beta$-unsaturated carbonyl system $(3,25,63)$. Various bioisosteres are used to mimic the spatial arrangement, electronic properties, and other physicochemical properties of these groups while retarding bioactivation. For example, the olefin could be replaced by a cyclopropyl group at the expense of a minor conformational change, or the carbonyl could be replaced with another group (e.g. difluoromethyl) whose inductive properties might limit oxidation of the olefin. These options are illustrated in Fig. 5.

\section{Metabolic Liabilities of Non-GSH Conjugates and Associated Intermediates}

Drug candidates that contain hydroxyl groups are prone to metabolic clearance via glucuronidation and sulfation, and, as a result, these compounds have relatively short durations of action and poor pharmacokinetics properties in vivo. The rate of conjugation depends on many factors and is related directly to the nucleophilicity of the phenolate. If the phenol is not required for activity and its nucleophilic character is not readily removed, then several options are available to improve metabolic stability while retaining the essential physicochemical properties of the molecule. Whereas an initial approach might be to replace the hydroxyl group with a methoxyl or other alkoxyl group, this substitution would be accompanied by a loss of H-bonding activity and may introduce unfavorable receptor interactions and/or an additional soft spot for CYP enzymes. To avoid these problems, a more common solution is to use an alkylsulfonamide as a bioisostere of the hydroxyl group, or a nitrogen-containing heterocycle (e.g. benzimidazolone, indole or indolone) in place of the entire phenol moiety (99) (Fig. 5). The $\mathrm{NH}$ moieties of these isosteres are comparable to phenols as both
Brønsted acids and H-bond donors and are not typical substrates for conjugation reactions during biotransformation.

Acyl glucuronides are identified increasingly as electrophilic intermediates capable of three reactions: intramolecular rearrangement, hydrolysis, and intermolecular transacylation with proteins leading to covalent adducts. Although several studies have been conducted to describe these processes in vitro, most have used experimental conditions designed to amplify bioactivation. The view that reactive acyl glucuronides may initiate toxicological or immune responses is widely believed to be plausible but also relies heavily on circumstantial evidence (100). To reduce the risk of metabolismrelated liabilities associated with acyl glucuronides, tetrazoles are often used to replace carboxylates. Tetrazoles are ionized at neutral $\mathrm{pH}$ and exhibit a planar structure, similar to carboxylates, but are also more permeable to membranes on account of improved lipophilicity (101). Amides, imides, and sulfonamides have also been explored as bioisosteric replacements for carboxylates (Fig. 5). Since the sulfonamide, acylsulfonamide, and sulfonylurea groups contain electronwithdrawing groups in proximity to a nitrogen atom, these isosteres are comparable to carboxylates in terms of acidity but have no potential to form acyl glucuronides. Additionally, these acid surrogates may offer a new tether for the synthetic modulation of pharmacological and/or pharmacokinetic properties $(102,103)$.

Several compounds are known to be metabolized to chemically reactive intermediates via $O$-sulfation. The instability of these compounds arises from the ability of the sulfate to act as a leaving group, facilitating $\mathrm{S}_{\mathrm{N}} 1$ via the formation of

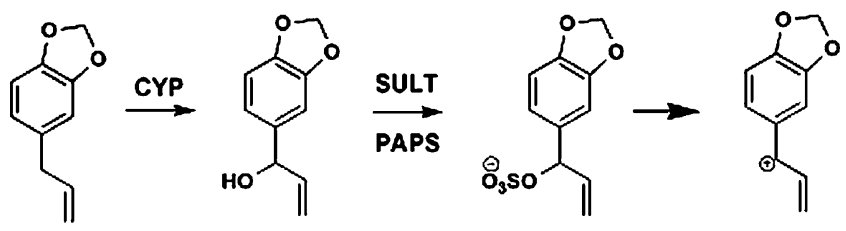

safrole

Fig. 10. Bioactivation of safrole via an unstable sulfate intermediate 
a strongly electrophilic cation. Reports have shown that sulfation is important in the activation of the toxicants safrole, $2^{\prime}, 3^{\prime}$-estragole, and 6-hydroxymethylbenzo[ $\left.a\right]$ pyrene (Fig. 10), and that sulfotransferase (SULT) inhibitors reduce the formation of macromolecular adducts (104). Studies using recombinant SULTs have demonstrated that bioactivation is usually mediated selectively by an individual SULT form. Bioactivation by SULTs also has a cyclic nature, since the reaction of a sulfuric acid ester with water may regenerate the hydroxyl precursor and feed a new cycle of activation (105). However, since the cofactor $3^{\prime}$-phosphoadenosyl-5'-phosphosulfate (PAPS) is depleted in cells more readily than uridine-5'diphosphate glucuronic acid (UDPGA), consumption of PAPS may eventually end this cycle and divert the metabolism of SULT substrates toward other biotransformation pathways. Until recently, studies of the toxicological effects associated with sulfate metabolites required such metabolites to be synthesized and tested separately, and were often confounded by the poor permeability of sulfate conjugates toward cell membranes. However, the heterologous expression of SULTs in cellular systems used during mutagenicity tests may represent a useful approach in resolving such experimental issues (105).

\section{CONCLUSION}

The successful integration of biotransformation studies into a drug-discovery workflow requires a few important elements. The first is a concerted effort between drug metabolism and medicinal chemistry groups to identify metabolism-related liabilities of key compounds in a timely manner, and to use this information effectively in managing the evolution of a lead series. The second is a willingness to continually evolve these practices in accordance with changing regulatory requirements, technological improvements, and the growing body of knowledge that lies at the interface between drug metabolism and toxicology. For the reasons outlined in this article, this dynamic paradigm is expected to assist in the advancement of drug candidates with a lower probability of metabolism-related liabilities and commensurately improved chances for success in the clinic. Ultimately, it is hoped that these changes will expedite the delivery of effective and safe new medicines to the patients who need them.

\section{ACKNOWLEDGEMENTS}

The authors thank Drs. W. Griffith Humphreys, Michael W. Sinz, and Sean Kim for helpful comments and suggestions.

\section{REFERENCES}

1. I. Kola, and J. Landis. Can the pharmaceutical industry reduce attrition rates? Nat. Rev. Drug Discov. 3:711-715 (2004).

2. A. E. Nassar, A. M. Kamel, and C. Clarimont. Improving the decision-making process in structural modification of drug candidates: reducing toxicity. Drug Discov. Today. 9:1055-1064 (2004).

3. A. S. Kalgutkar, I. Gardner, R. S. Obach, et al. A comprehensive listing of bioactivation pathways of organic functional groups. Curr. Drug Metab. 6:161-225 (2005).
4. K. Park, D. P. Williams, D. J. Naisbitt, N. R. Kitteringham, and $\mathrm{M}$. Pirmohamed. Investigation of toxic metabolites during drug development. Toxicol. Appl. Pharmacol. 207:425-434 (2005).

5. R. J. Riley, K. Grime, and R. Weaver. Time-dependent CYP inhibition. Expert. Opin. Drug Metab. Toxicol. 3:51-66 (2007).

6. R. Silverman. Mechanism-based Enzyme Inactivation, CRC Press, Boca Raton, FL, USA, 1998.

7. F. Ghanbari, K. Rowland-Yeo, J. C. Bloomer, et al. A critical evaluation of the experimental design of studies of mechanism based enzyme inhibition, with implications for in vitro-in vivo extrapolation. Curr. Drug Metab. 7:315-334 (2006).

8. R. S. Obach, R. L. Walsky, and K. Venkatakrishnan. Mechanism-based inactivation of human cytochrome p450 enzymes and the prediction of drug-drug interactions. Drug Metab. Dispos. 35:246-255 (2007).

9. K. Venkatakrishnan, and R. S. Obach. Drug-drug interactions via mechanism-based cytochrome P450 inactivation: points to consider for risk assessment from in vitro data and clinical pharmacologic evaluation. Curr. Drug Metab. 8:449-462 (2007).

10. J. Uetrecht. Idiosyncratic drug reactions: current understanding. Annu. Rev. Pharmacol. Toxicol. 47:513-539 (2007).

11. J. M. Shenton, M. Teranishi, M. S. Abu-Asab, J. A. Yager, and J. P. Uetrecht. Characterization of a potential animal model of an idiosyncratic drug reaction: nevirapine-induced skin rash in the rat. Chem. Res. Toxicol. 16:1078-1089 (2003).

12. W. Lu, and J. P. Uetrecht. Possible bioactivation pathways of lamotrigine. Drug Metab. Dispos. 35:1050-1056 (2007).

13. J. Uetrecht. Evaluation of which reactive metabolite, if any, is responsible for a specific idiosyncratic reaction. Drug Metab. Rev. 38:745-753 (2006).

14. J. L. Bolton, M. A. Trush, T. M. Penning, G. Dryhurst, and T. J. Monks. Role of quinones in toxicology. Chem. Res. Toxicol. 13:135-160 (2000).

15. D. C. Thompson, K. Perera, E. S. Krol, and J. L. Bolton. oMethoxy-4-alkylphenols that form quinone methides of intermediate reactivity are the most toxic in rat liver slices. Chem. Res. Toxicol. 8:323-327 (1995).

16. J. N. Commandeur, G. J. Stijntjes, and N. P. Vermeulen. Enzymes and transport systems involved in the formation and disposition of glutathione $S$-conjugates. Role in bioactivation and detoxication mechanisms of xenobiotics. Pharmacol. Rev. 47:271-330 (1995).

17. W. G. Humphreys, and S. E. Unger. Safety assessment of drug metabolites: Characterization of chemically stable metabolites. Chem. Res. Toxicol. 19:1564-1569 (2006).

18. T. A. Baillie, P. G. Pearson, M. S. Rashed, and W. N. Howald. The use of mass spectrometry in the study of chemicallyreactive drug metabolites. Application of MS/MS and LC/MS to the analysis of glutathione- and related $S$-linked conjugates of $N$-methylformamide. J. Pharm. Biomed. Anal. 7:1351-1360 (1989).

19. B. M. Johnson, S. X. Qiu, S. Zhang, et al. Identification of novel electrophilic metabolites of piper methysticum Forst (Kava). Chem. Res. Toxicol. 16:733-740 (2003).

20. J. Gan, T. W. Harper, M. M. Hsueh, Q. Qu, and W. G. Humphreys. Dansyl glutathione as a trapping agent for the quantitative estimation and identification of reactive metabolites. Chem. Res. Toxicol. 18:896-903 (2005).

21. J. R. Soglia, S. P. Harriman, S. Zhao, et al. The development of a higher throughput reactive intermediate screening assay incorporating micro-bore liquid chromatography-micro-electrospray ionization-tandem mass spectrometry and glutathione ethyl ester as an in vitro conjugating agent. J. Pharm. Biomed. Anal. 36:105-116 (2004).

22. A. Mutlib, W. Lam, J. Atherton, H. Chen, P. Galatsis, and W. Stolle. Application of stable isotope labeled glutathione and rapid scanning mass spectrometers in detecting and characterizing reactive metabolites. Rapid Commun. Mass Spectrom. 19:3482-3492 (2005).

23. J. W. Gorrod, and G. Aislaitner. The metabolism of alicyclic amines to reactive iminium ion intermediates. Eur. J. Drug Metab. Pharmacokinet. 19:209-217 (1994). 
24. N. Masubuchi, C. Makino, and N. Murayama. Prediction of in vivo potential for metabolic activation of drugs into chemically reactive intermediate: correlation of in vitro and in vivo generation of reactive intermediates and in vitro glutathione conjugate formation in rats and humans. Chem. Res. Toxicol. 20:455-464 (2007).

25. D. C. Evans, A. P. Watt, D. A. Nicoll-Griffith, and T. A. Baillie. Drug-protein adducts: an industry perspective on minimizing the potential for drug bioactivation in drug discovery and development. Chem. Res. Toxicol. 17:3-16 (2004).

26. A. S. Kalgutkar, R. S. Obach, and T. S. Maurer. Mechanismbased inactivation of cytochrome P450 enzymes: chemical mechanisms, structure-activity relationships and relationship to clinical drug-drug interactions and idiosyncratic adverse drug reactions. Curr. Drug Metab. 8:407-447 (2007).

27. D. R. Jones, J. C. Gorski, M. A. Hamman, B. S. Mayhew, S. Rider, and S. D. Hall. Diltiazem inhibition of cytochrome P-450 $3 \mathrm{~A}$ activity is due to metabolite intermediate complex formation. J. Pharmacol. Exp. Ther. 290:1116-1125 (1999).

28. E. Fontana, P. M. Dansette, and S. M. Poli. Cytochrome p450 enzymes mechanism based inhibitors: common sub-structures and reactivity. Curr. Drug Metab. 6:413-454 (2005).

29. H. L. Lin, U. M. Kent, and P. F. Hollenberg. Mechanism-based inactivation of cytochrome P450 3A4 by 17 alpha-ethynylestradiol: evidence for heme destruction and covalent binding to protein. $J$. Pharmacol. Exp. Ther. 301:160-167 (2002).

30. B. S. Mayhew, D. R. Jones, and S. D. Hall. An in vitro model for predicting in vivo inhibition of cytochrome P450 3A4 by metabolic intermediate complex formation. Drug Metab. Dispos. 28:1031-1037 (2000)

31. C. S. Ernest 2nd, S. D. Hall, and D. R. Jones. Mechanism-based inactivation of CYP3A by HIV protease inhibitors. J. Pharmacol. Exp. Ther. 312:583-591 (2005).

32. B. Ma, T. Prueksaritanont, and J. H. Lin. Drug interactions with calcium channel blockers: possible involvement of metaboliteintermediate complexation with CYP3A. Drug Metab. Dispos. 28:125-130 (2000)

33. O. M. Bakke, W. M. Wardell, and L. Lasagna. Drug discontinuations in the United Kingdom and the United States, 1964 to 1983: issues of safety. Clin. Pharmacol. Ther. 35:559-567 (1984).

34. J. Wang, M. Davis, F. Li, F. Azam, J. Scatina, and R. Talaat. A novel approach for predicting acyl glucuronide reactivity via Schiff base formation: development of rapidly formed peptide adducts for LC/MS/MS measurements. Chem. Res. Toxicol. 17:1206-1216 (2004).

35. G. S. Walker, J. Atherton, J. Bauman, et al. Determination of degradation pathways and kinetics of acyl glucuronides by NMR spectroscopy. Chem. Res. Toxicol. 20:876-886 (2007).

36. E. Skordi, I. D. Wilson, J. C. Lindon, and J. K. Nicholson. Kinetic studies on the intramolecular acyl migration of beta-1$O$-acyl glucuronides: application to the glucuronides of $(R)$ - and $(S)$-ketoprofen, $(R)$ - and $(S)$-hydroxy-ketoprofen metabolites, and tolmetin by 1H-NMR spectroscopy. Xenobiotica. 35:715725 (2005)

37. S. J. Vanderhoeven, J. C. Lindon, J. Troke, J. K. Nicholson, and I. D. Wilson. NMR spectroscopic studies of the transacylation reactivity of ibuprofen 1-beta- $O$-acyl glucuronide. J. Pharm. Biomed. Anal. 41:1002-1006 (2006).

38. M. R. Anari, R. I. Sanchez, R. Bakhtiar, R. B. Franklin, and T. A. Baillie. Integration of knowledge-based metabolic predictions with liquid chromatography data-dependent tandem mass spectrometry for drug metabolism studies: application to studies on the biotransformation of indinavir. Anal. Chem. 76:823-832 (2004).

39. A. Rostami-Hodjegan, and G. T. Tucker. Simulation and prediction of in vivo drug metabolism in human populations from in vitro data. Nat. Rev. Drug Discov. 6:140-148 (2007).

40. J. C. Madden, and M. T. Cronin. Structure-based methods for the prediction of drug metabolism. Expert. Opin. Drug Metab. Toxicol. 2:545-557 (2006).

41. S. Ekins, S. Andreyev, A. Ryabov, et al. Computational prediction of human drug metabolism. Expert. Opin. Drug Metab. Toxicol. 1:303-324 (2005).
42. S. S. De Buck, V. K. Sinha, L. A. Fenu, R. A. Gilissen, C. E. Mackie, and M. J. Nijsen. The prediction of drug metabolism, tissue distribution, and bioavailability of 50 structurally diverse compounds in rat using mechanism-based absorption, distribution, and metabolism prediction tools. Drug Metab. Dispos. 35:649-659 (2007).

43. G. Cruciani, E. Carosati, B. De Boeck, et al. MetaSite: understanding metabolism in human cytochromes from the perspective of the chemist. J. Med. Chem. 48:6970-6979 (2005).

44. R. P. Sheridan, K. R. Korzekwa, R. A. Torres, and M. J. Walker. Empirical regioselectivity models for human cytochromes P450 3A4, 2D6, and 2C9. J. Med. Chem. 50:31733184 (2007).

45. J. Langowski, and A. Long. Computer systems for the prediction of xenobiotic metabolism. Adv. Drug Deliv. Rev. 54:407-415 (2002).

46. P. R. Ortiz de Montellano, and J. M. Matthews. Autocatalytic alkylation of the cytochrome P-450 prosthetic haem group by 1 aminobenzotriazole. Isolation of an NN-bridged benzyne-protoporphyrin IX adduct. Biochem. J. 195:761-764 (1981).

47. S. K. Balani, T. Zhu, T. J. Yang, Z. Liu, B. He, and F. W. Lee. Effective dosing regimen of 1-aminobenzotriazole for inhibition of antipyrine clearance in rats, dogs, and monkeys. Drug Metab. Dispos. 30:1059-1062 (2002).

48. S. K. Balani, P. Li, J. Nguyen, et al. Effective dosing regimen of 1-aminobenzotriazole for inhibition of antipyrine clearance in guinea pigs and mice using serial sampling. Drug Metab. Dispos. 32:1092-1095 (2004).

49. D. P. Williams, D. J. Antoine, P. J. Butler, et al. The metabolism and toxicity of furosemide in the wistar rat and CD-1 mouse: a chemical and biochemical definition of the toxicophore. $J$. Pharmacol. Exp. Ther. 322:1208-1220 (2007).

50. C. M. Henesey, and P. J. Harvison. Renal damage, metabolism and covalent binding following administration of the nephrotoxicant $N$-(3,5-dichlorophenyl)succinimide (NDPS) to male Fischer 344 rats. Toxicology. 170:187-200 (2002).

51. K. L. Davis-Bruno, and A. Atrakchi. A regulatory perspective on issues and approaches in characterizing human metabolites. Chem. Res. Toxicol. 19:1561-1563 (2006).

52. W. Li, D. Zhang, L. Wang, et al. Biotransformation of carbon14-labeled muraglitazar in male mice: interspecies difference in metabolic pathways leading to unique metabolites. Drug Metab. Dispos. 34:807-820 (2006).

53. K. Matsui, M. Mishima, Y. Nagai, T. Yuzuriha, and T. Yoshimura. Absorption, distribution, metabolism, and excretion of donepezil (Aricept) after a single oral administration to Rat. Drug Metab. Dispos. 27:1406-1414 (1999).

54. S. J. Roffey, R. S. Obach, J. I. Gedge, and D. A. Smith. What is the objective of the mass balance study? A retrospective analysis of data in animal and human excretion studies employing radiolabeled drugs. Drug Metab. Rev. 39:17-43 (2007).

55. D. Pereg, N. Tampal, P. Espandiari, and L. W. Robertson. Distribution and macromolecular binding of benzo[a]pyrene and two polychlorinated biphenyl congeners in female mice. Chem. Biol. Interact. 137:243-258 (2001).

56. Y. Hsieh, J. Chen, and W. A. Korfmacher. Mapping pharmaceuticals in tissues using MALDI imaging mass spectrometry. $J$. Pharmacol. Toxicol. Methods. 55:193-200 (2007).

57. M. L. Reyzer, Y. Hsieh, K. Ng, W. A. Korfmacher, and R. M. Caprioli. Direct analysis of drug candidates in tissue by matrixassisted laser desorption/ionization mass spectrometry. J. Mass Spectrom. 38:1081-1092 (2003).

58. M. L. Reyzer, and R. M. Caprioli. MALDI-MS-based imaging of small molecules and proteins in tissues. Feb. Curr. Opin. Chem. Biol. 11:29-35 (2007).

59. A. M. Yu. Small interfering RNA in drug metabolism and transport. Curr. Drug Metab. 8:700-708 (2007).

60. T. M. Fountaine, and R. Wade-Martins. RNA interferencemediated knockdown of alpha-synuclein protects human dopaminergic neuroblastoma cells from MPP $(+)$ toxicity and reduces dopamine transport. J. Neurosci. Res. 85:351-363 (2007). 
61. F. J. Gonzalez. CYP3A4 and pregnane X receptor humanized mice. J. Biochem. Mol. Toxicol. 21:158-162 (2007).

62. F. J. Gonzalez, and A. M. Yu. Cytochrome P450 and xenobiotic receptor humanized mice. Annu. Rev. Pharmacol. Toxicol. 46:41-64 (2006).

63. F. P. Guengerich. Common and uncommon cytochrome P450 reactions related to metabolism and chemical toxicity. Chem. Res. Toxicol. 14:611-650 (2001).

64. N. Castagnoli Jr., J. M. Rimoldi, J. Bloomquist, and K. P. Castagnoli. Potential metabolic bioactivation pathways involving cyclic tertiary amines and azaarenes. Chem. Res. Toxicol. 10:924-940 (1997).

65. B. M. Moore, F. C. Seaman, R. T. Wheelhouse, and L. Hurley. Mechanism for the catalytic activation of ecteinascidin 743 and its subsequent alkylation of guanine N2. J. Am. Chem. Soc. 120:2490-2491 (1998).

66. G. C. Hill, T. P. Wunz, N. E. MacKenzie, P. R. Gooley, and W. A. Remers. Computer simulation of the binding of naphthyridinomycin and cyanocycline A to DNA. J. Med. Chem. 34:2079-2088 (1991).

67. S. M. Cutts, A. Nudelman, A. Rephaeli, and D. R. Phillips. The power and potential of doxorubicin-DNA adducts. IUBMB Life. 57:73-81 (2005).

68. M. A. Moufarij, S. M. Cutts, G. M. Neumann, K. Kimura, and D. R. Phillips. Barminomycin functions as a potent preactivated analogue of adriamycin. Chem. Biol. Interact. 138:137-153 (2001).

69. D. E. Williams, R. L. Reed, B. Kedzierski, G. A. Dannan, F. P. Guengerich, and D. R. Buhler. Bioactivation and detoxication of the pyrrolizidine alkaloid senecionine by cytochrome P-450 enzymes in rat liver. Drug Metab. Dispos. 17:387-392 (1989).

70. Pyrrolizidine Alkaloids. In International Programme on Chemical Safety I, (ed.), Environmental Health Criteria. Vol 80: UN Environment Programme, International Labour Organization, WHO, 1988.

71. S. Kumar, K. Kassahun, R. A. Tschirret-Guth, K. Mitra, and T. A. Baillie. Minimizing metabolic activation during pharmaceutical lead optimization: Progress, knowledge gaps and future directions. Curr. Opin. Drug Discov. Devel. 11:43-52 (2008).

72. N. A. Thornberry, and A. E. Weber. Discovery of JANUVIA (Sitagliptin), a selective dipeptidyl peptidase IV inhibitor for the treatment of type 2 diabetes. Curr. Top Med. Chem. 7:557568 (2007).

73. R. C. Garner, C. N. Martin, and D. B. Clayson. Carcinogenic aromatic amines and related compounds. In C. E. Searle (ed.), Chemical Carcinogens, Vol 1, American Chemical Society, Washington, DC, USA, 1982, pp. 175-276.

74. C. R. Jones, and G. Sabbioni. Identification of DNA adducts using HPLC/MS/MS following in vitro and in vivo experiments with arylamines and nitroarenes. Chem. Res. Toxicol. 16:12511263 (2003).

75. X. Chen, A. Christopher, J. P. Jones, et al. Crystal structure of the $\mathrm{F} 87 \mathrm{~W} / \mathrm{Y} 96 \mathrm{~F} / \mathrm{V} 247 \mathrm{~L}$ mutant of cytochrome P-450cam with 1,3,5-trichlorobenzene bound and further protein engineering for the oxidation of pentachlorobenzene and hexachlorobenzene. J. Biol. Chem. 277:37519-37526 (2002).

76. S. J. Roffey, S. Cole, P. Comby, et al. The disposition of voriconazole in mouse, rat, rabbit, guinea pig, dog, and human. Drug Metab. Dispos. 31:731-741 (2003).

77. H. Chen, J. Shockcor, W. Chen, R. Espina, L. S. Gan, and A. E. Mutlib. Delineating novel metabolic pathways of DPC 963, a non-nucleoside reverse transcriptase inhibitor, in rats. Characterization of glutathione conjugates of postulated oxirene and benzoquinone imine intermediates by LC/MS and LC/NMR. Chem. Res. Toxicol. 15:388-399 (2002).

78. D. K. Dalvie, A. S. Kalgutkar, S. C. Khojasteh-Bakht, R. S. Obach, J. P. O, and Donnell. Biotransformation reactions of five-membered aromatic heterocyclic rings. Chem. Res. Toxicol. 15:269-299 (2002).

79. K. L. Kunze, W. L. Nelson, E. D. Kharasch, K. E. Thummel, and N. Isoherranen. Stereochemical aspects of itraconazole metabolism in vitro and in vivo. Drug Metab. Dispos. 34:583590 (2006).

80. J. T. Pearson, J. J. Hill, J. Swank, N. Isoherranen, K. L. Kunze, and W. M. Atkins. Surface plasmon resonance analysis of antifungal azoles binding to CYP3A4 with kinetic resolution of multiple binding orientations. Biochemistry. 45:6341-6353 (2006).

81. C. W. Locuson, J. M. Hutzler, and T. S. Tracy. Visible spectra of type II cytochrome P450-drug complexes: evidence that "incomplete" heme coordination is common. Drug Metab. Dispos. 35:614-622 (2007)

82. J. M. Hutzler, R. C. Steenwyk, E. B. Smith, G. S. Walker, and L. C. Wienkers. Mechanism-based inactivation of cytochrome P450 2D6 by 1-[(2-ethyl-4-methyl-1H-imidazol-5-yl)methyl]- 4-[4(trifluoromethyl)-2-pyridinyl]piperazine: kinetic characterization and evidence for apoprotein adduction. Chem. Res. Toxicol. 17:174-184 (2004).

83. T. J. Monks, and D. C. Jones. The metabolism and toxicity of quinones, quinonimines, quinone methides, and quinonethioethers. Curr. Drug Metab. 3:425-438 (2002).

84. B. Kalyanaraman, P. I. Premovic, and R. C. Sealy. Semiquinone anion radicals from addition of amino acids, peptides, and proteins to quinones derived from oxidation of catechols and catecholamines. An ESR spin stabilization study. J. Biol. Chem. 262:11080-11087 (1987).

85. S. S. Lau, B. A. Hill, R. J. Highet, and T. J. Monks. Sequential oxidation and glutathione addition to 1,4-benzoquinone: correlation of toxicity with increased glutathione substitution. Mol. Pharmacol. 34:829-836 (1988).

86. K. G. Eckert, P. Eyer, J. Sonnenbichler, and I. Zetl. Activation and detoxication of aminophenols. III. Synthesis and structural elucidation of various glutathione addition products to 1,4benzoquinone. Xenobiotica. 20:351-361 (1990).

87. P. E. Weller, and R. P. Hanzlik. Isolation of $S$-(bromophenyl) cysteine isomers from liver proteins of bromobenzene-treated rats. Chem. Res. Toxicol. 4:17-20 (1991).

88. T. J. Monks, R. P. Hanzlik, G. M. Cohen, D. Ross, and D. G Graham. Quinone chemistry and toxicity. Toxicol. Appl. Pharmacol. 112:2-16 (1992).

89. A. Chenna, B. Hang, B. Rydberg, et al. The benzene metabolite $p$-benzoquinone forms adducts with DNA bases that are excised by a repair activity from human cells that differs from an ethenoadenine glycosylase. Proc. Natl. Acad. Sci. U. S. A. 92:5890-5894 (1995).

90. Q. Zhou, and S. E. Rokita. A general strategy for targetpromoted alkylation in biological systems. Proc. Natl. Acad. Sci. U. S. A. 100:15452-15457 (2003).

91. T. Atsumi, S. Fujisawa, and K. Tonosaki. A comparative study of the antioxidant/prooxidant activities of eugenol and isoeugenol with various concentrations and oxidation conditions. Toxicol. In Vitro. 19:1025-1033 (2005).

92. W. G. Lai, N. Zahid, and J. P. Uetrecht. Metabolism of trimethoprim to a reactive iminoquinone methide by activated human neutrophils and hepatic microsomes. J. Pharmacol. Exp. Ther. 291:292-299 (1999).

93. R. Singh, M. V. Silva Elipe, P. G. Pearson, et al. Metabolic activation of a pyrazinone-containing thrombin inhibitor. Evidence for novel biotransformation involving pyrazinone ring oxidation, rearrangement, and covalent binding to proteins. Chem. Res. Toxicol. 16:198-207 (2003).

94. W. Bicker, M. Lammerhofer, D. Genser, H. Kiss, and W. Lindner. A case study of acute human chlorpyrifos poisoning: novel aspects on metabolism and toxicokinetics derived from liquid chromatography-tandem mass spectrometry analysis of urine samples. Toxicol. Lett. 159:235-251 (2005).

95. K. A. Koeplinger, Z. Zhao, T. Peterson, et al. Activated sulfonamides are cleaved by glutathione-S-transferases. Drug Metab. Dispos. 27:986-991 (1999).

96. Z. Zhao, K. A. Koeplinger, T. Peterson, et al. Mechanism, structure-activity studies, and potential applications of glutathione $S$-transferase-catalyzed cleavage of sulfonamides. Drug Metab. Dispos. 27:992-998 (1999). 
97. C. W. Conroy, H. Schwam, and T. H. Maren. The nonenzymatic displacement of the sulfamoyl group from different classes of aromatic compounds by glutathione and cysteine. Drug Metab. Dispos. 12:614-618 (1984).

98. M. Fukuoka, M. Satoh, and A. Tanaka. Metabolism of 2thiobenzothiazoles in the rat. Urinary, fecal and biliary metabolites of 2-benzothiazyl sulfenamides. Arch. Toxicol. 70:1-9 (1995).

99. X. Chen, and W. Wang. The use of bioisosteric groups in lead optimization. In M. Desai (ed.), Ann. Rep. Med. Chem. Vol 38, American Chemical Society, Washington DC, USA, 2003, pp. 333-346.

100. M. J. Bailey, and R. G. Dickinson. Acyl glucuronide reactivity in perspective: biological consequences. Chem. Biol. Interact. 145:117-137 (2003).
101. R. J. Herr. 5-Substituted-1H-tetrazoles as carboxylic acid isosteres: medicinal chemistry and synthetic methods. Bioorg. Med. Chem. 10:3379-3393 (2002).

102. M. Gallant, M. C. Carriere, A. Chateauneuf, et al. Structureactivity relationship of biaryl acylsulfonamide analogues on the human EP(3) prostanoid receptor. Bioorg. Med. Chem. Lett. 12:2583-2586 (2002).

103. D. E. Uehling, K. H. Donaldson, D. N. Deaton, et al. Synthesis and evaluation of potent and selective beta(3) adrenergic receptor agonists containing acylsulfonamide, sulfonylsulfonamide, and sulfonylurea carboxylic acid isosteres. J. Med. Chem. 45:567-583 (2002).

104. J. A. Miller. Sulfonation in chemical carcinogenesis-history and present status. Chem. Biol. Interact. 92:329-341 (1994).

105. H. Glatt. Sulfotransferases in the bioactivation of xenobiotics. Chem. Biol. Interact. 129:141-170 (2000). 